\title{
A chromosome-scale reference genome of Lobularia maritima, an ornamental plant with high stress tolerance
}

\author{
Li Huang ${ }^{1}$, Yazhen Ma ${ }^{1}$, Jiebei Jiang ${ }^{1}$, Ting Li ${ }^{1}$, Wenjie Yang ${ }^{1}$, Lei Zhang ${ }^{1}$, Lei W ${ }^{1}$, Landi Feng ${ }^{1}$, Zhenxiang Xi', \\ Xiaoting $\mathrm{Xu}^{1}$, Jianquan Liu $\mathbb{1}^{1,2}$ and Quanjun Hu $\mathbb{B}^{1}$
}

\begin{abstract}
Lobularia maritima (L.) Desv. is an ornamental plant cultivated across the world. It belongs to the family Brassicaceae and can tolerate dry, poor and contaminated habitats. Here, we present a chromosome-scale, high-quality genome assembly of L. maritima based on integrated approaches combining Illumina short reads and $\mathrm{Hi}-\mathrm{C}$ chromosome conformation data. The genome was assembled into 12 pseudochromosomes with a $197.70 \mathrm{Mb}$ length, and it includes 25,813 protein-coding genes. Approximately $41.94 \%$ of the genome consists of repetitive sequences, with abundant long terminal repeat transposable elements. Comparative genomic analysis confirmed that L. maritima underwent a species-specific whole-genome duplication (WGD) event 22.99 million years ago. We identified $\sim 1900$ species-specific genes, 25 expanded gene families, and 50 positively selected genes in L. maritima. Functional annotations of these genes indicated that they are mainly related to stress tolerance. These results provide new insights into the stress tolerance of L. maritima, and this genomic resource will be valuable for further genetic improvement of this important ornamental plant.
\end{abstract}

\section{Introduction}

Whole-genome duplication (WGD), or polyploidy, has had a strong influence on the evolution of the tree of life, and it seems to have occurred in the evolutionary history of most plant species $^{1,2}$, especially in angiosperms ${ }^{3}$. WGDs have been found in most angiosperm families with abundant species, including Brassicaceae, Poaceae, Asteraceae, Solanaceae, Fabaceae and Orchidaceae ${ }^{4-11}$. Previous studies suggested that WGDs can strengthen the adaptation of plants to environmental challenges ${ }^{12}$ because of genomic reorganization and novelties. Through subfunctionalization or reciprocal loss of duplicated genes in differentiated populations of an

Correspondence: Quanjun Hu (huquanjun@gmail.com)

${ }^{1}$ Key Laboratory of Bio-Resource and Eco-Environment of Ministry of Education, College of Life Sciences, Sichuan University, 610065 Chengdu, China ${ }^{2}$ State Key Laboratory of Grassland Agro-Ecosystem, Institute of Innovation Ecology, Lanzhou University, Lanzhou, China

These authors contributed equally: Li Huang, Yazhen Ma ancestral species, WGDs can also promote reproductive isolation and thus facilitate speciation ${ }^{13}$. Brassicaceae (also known as Cruciferae), a monophyletic group distributed worldwide, has been highly diversified by complicated WGD events and subsequent evolution, with $\sim 350$ genera and 4000 species $^{14,15}$. It contains many important crops (e.g., cabbage, rapeseed and mustard) that have been domesticated for food, biofuels, and ornamentals ${ }^{16}$. The well-known model organism Arabidopsis thaliana, which is of paramount importance in studies of the development, gene expression and genome evolution of flowering plants, is also a member of this family ${ }^{17,18}$. Analyses of the A. thaliana genome have provided clear evidence that three ancient WGD events $(\gamma, \beta$ and $\alpha)$, occurred in its evolutionary history. The oldest WGD event, the At- $\gamma$ event, was related to the diversification of eudicots and perhaps all angiosperms $^{19-21}$. The At- $\beta$ event postdated the Brassicaceae-Caricaceae divergence $\sim 70$ million years ago

\section{(c) The Author(s) 2020}

(c) (i) Open Access This article is licensed under a Creative Commons Attribution 4.0 International License, which permits use, sharing, adaptation, distribution and reproduction cc) in any medium or format, as long as you give appropriate credit to the original author(s) and the source, provide a link to the Creative Commons license, and indicate if changes were made. The images or other third party material in this article are included in the article's Creative Commons license, unless indicated otherwise in a credit line to the material. If material is not included in the article's Creative Commons license and your intended use is not permitted by statutory regulation or exceeds the permitted use, you will need to obtain permission directly from the copyright holder. To view a copy of this license, visit http://creativecommons.org/licenses/by/4.0/. 
$(\text { Mya })^{22,23}$. However, the At- $\alpha$ event was specific to the Brassicaceae family ${ }^{19}$, occurring $\sim 40 \mathrm{Mya}^{24}$. In addition, independent WGDs more recent than the Neogene may have promoted the colonization of harsh environments by Brassicaceae taxa by increasing their stress tolerance and conferring high adaptability ${ }^{25,26}$. However, detailed investigation of WGDs in numerous genera present in arid habitats is still badly needed ${ }^{27,28}$.

Lobularia maritima (L.) Desv., commonly known as sweet alyssum, is a perennial and diploid $(2 n=24)$ herbaceous plant of the family Brassicaceae. This ornamental plant naturally occurs in the western Mediterranean region and has been widely cultivated since its domestication ${ }^{29,30}$. Its flowers range in color from pale violet to deep purple ${ }^{31}$. In addition to tolerating dry and poor habitats, L. maritima is recognized as a nickel hyperaccumulator that can remove heavy metals from contaminated soils ${ }^{32}$. As a facultative halophyte closely related to Arabidopsis thaliana, L. maritima seems to be an ideal model for revealing the molecular mechanisms underlying plant tolerance to drought and salt stress ${ }^{33}$. However, studies of L. maritima have focused mainly on its cultivation, management and rapid propagation in vitro ${ }^{29}$.

In this study, we report a chromosome-scale assembly of the L. maritima genome anchored on 12 pseudochromosomes. We further identified a recent L. maritimaspecific WGD event that occurred after the Brassicaceaespecific At- $\alpha$ event using comparative and evolutionary analyses. We also revealed numerous genomic changes by which L. maritima has adapted to harsh habitats.

\section{Results}

\section{Genome sequencing and assembly}

Samples for genome sequencing were obtained from an L. maritima seedling with purple flowers (Fig. 1a). We obtained $59.77 \mathrm{~Gb}$ of clean reads with various insert sizes and $22.31 \mathrm{~Gb}$ of $\mathrm{Hi}-\mathrm{C}$ clean reads ( 112.89-fold coverage) after Illumina sequencing and quality control (Supplementary Table 1). Two methods were employed to estimate the genome size of $L$. maritima. First, we determined the L. maritima genome size to be $225 \mathrm{Mb}$ using flow cytometry with $A$. thaliana as the external control (Supplementary Fig. 1). Second, we used $k$-merbased statistics ${ }^{34}$, and the genome size was calculated to be $264 \mathrm{Mb}$ (Supplementary Fig. 2).

Based on the clean reads, a de novo genome was assembled with a $197.70 \mathrm{Mb}$ length. We further anchored this genome on 12 pseudochromosomes (Fig. 1b and Table 1 and Supplementary Fig. 3). We then evaluated the completeness of this genome using BUSCO v4.1.2 ${ }^{35}$ and found that $99 \%$ of the single-copy orthologs were intact (Supplementary Table 2), suggesting the high quality of the assembled genome.

\section{Genome annotation}

To predict protein-coding sequences, we combined de novo and homology- and transcriptome-based methods. We predicted 25,813 complete protein-coding genes. Gene length and the number of exons of these proteincoding genes were 2431 base pairs (bp) and 5.46 exons, respectively, on average (Table 1). In our assembly, $97.99 \%(25,295$ of 25,813$)$ of the genes were annotated on 12 pseudochromosomes, and only $2.01 \%$ (518 of 25,813 ) were located on scaffolds. The Circos v0.69 (http://circos. ca) was used to visualize the collinearity blocks between $L$. maritima and Capsella rubella, gene density, Copia density, and Gypsy density on individual chromosomes (Fig. 1c). Among the 25,813 predicted genes, $81.30 \%$ and 95.71\% had homologs in the Swiss-Prot ${ }^{36}$ and TrEMBL ${ }^{36}$ databases, respectively. Additionally, we annotated $95.15 \%, 80.65 \%$, and $36.55 \%$ of the genes using the InterPro $^{37}$, Gene Ontology (GO) ${ }^{38}$ and Kyoto Encyclopedia of Genes and Genomes (KEGG) ${ }^{39}$ databases, respectively (Supplementary Table 3 ). In addition, $41.94 \%$ $(83 \mathrm{Mb})$ of the assembled L. maritima genome comprised repetitive sequences (Supplementary Table 4). Of these repetitive sequences, long terminal repeat (LTR) retrotransposons were the most frequent, spanning $14.24 \%$ of the assembled genome with $13.23 \%$ intact LTR retrotransposons. The other common repetitive sequences were DNA transposons (10.06\%), Tandem Repeats (8.56\%) and LINEs (5.65\%) (Supplementary Tables 4 and 5). To analyze the evolutionary dynamics of these LTRs, we estimated their insertion dates in four related species (A. thaliana, Arabidopsis lyrata, C. rubella and L. maritima). The recent insertions in A. lyrata may have contributed to its relatively large genome size $(207 \mathrm{Mb})$. Similarly, L. maritima had more recent insertions than $A$. thaliana and C. rubella (Fig. $1 \mathrm{~d}$ and Supplementary Table 5). Diverse genetic changes can be caused by transposable elements (including LTR retrotransposons), which might have promoted lineage-specific diversification and adaptation $^{40}$. This may partly contribute to the tolerance of $L$. maritima to arid habitats. However, the L. maritima genome contained a similar number of transcription factors (1799) as the other closely related Brassicaceae species (Supplementary Table 6, all transcription factor data for other species were downloaded from http://www. transcriptionfactor.org).

\section{Comparative genomic analyses and WGD analyses}

Using the ColinearScan v1.0.1 ${ }^{41}$ program and MCScanX $\mathrm{v}^{42}$ package, the protein sequences of L. maritima were compared to those of the diploid C. rubella, which has not been affected by a recent WGD event, to identify the collinear blocks in the genomes. The whole-genome alignments showed high collinearity and conservation, and several collinear regions almost completely spanned 


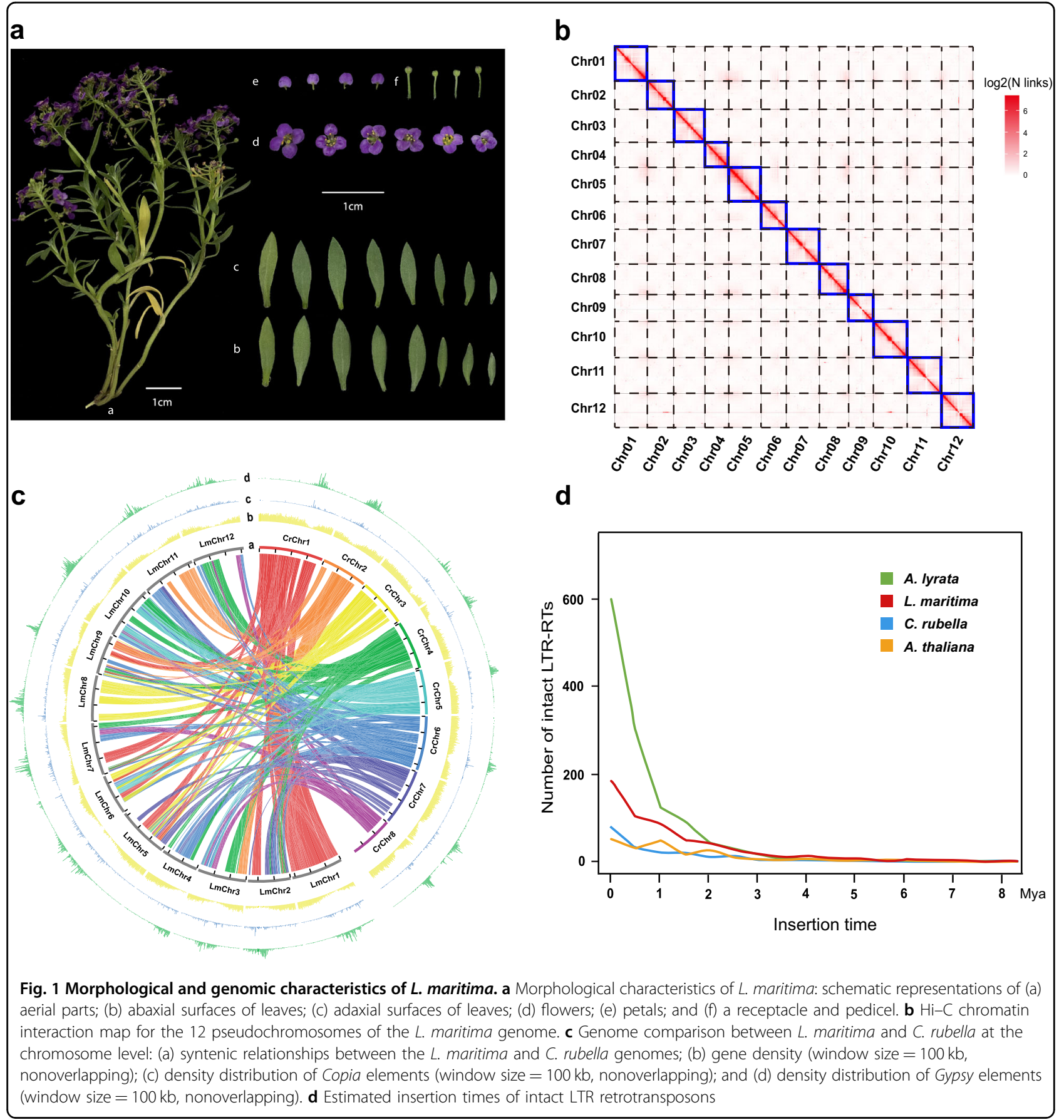

chromosomes of the two species (Fig. 2). It is worth noting that each chromosome or chromosomal region in C. rubella was represented on multiple independent chromosomes in the L. maritima genome after the Brassicaceae-specific At- $\alpha$ WGD event, suggesting that the L. maritima genome experienced a specific WGD event.

Furthermore, we determined the karyotype of $L$. maritima using previously reported methods ${ }^{28,43}$
(Supplementary Fig. 4) and recovered two sets of conserved genomic blocks ${ }^{44,45}$. However, the patterns of genomic blocks suggested that L. maritima experienced many postpolyploid diploidization events and a reduction in chromosome number. We also analyzed the gene retention rates of the two subgenomes in each genomic block with the $C$. rubella genome as the reference. The results showed that the two subgenomes retained similar numbers of genes (Supplementary Table 7). We also 
assessed the absence or presence of genome dominance by examining the expression levels of each pair of duplicated genes with high confidence. Based on RNA-seq data from flower, leaf, and stem tissues, we failed to find any evidence of biased expression in each genomic block between the two subgenomes (Supplementary Table 8 ).

Table 1 Assembly and annotation statistics of the L. maritima genome

\begin{tabular}{ll}
\hline Genome feature & Value \\
\hline Estimated genome size (Mb) & 264 \\
Total scaffold number & 27,734 \\
Total length (bp) & $197,688,650$ \\
Total length of chromosomes (bp) & $174,586,151$ \\
Longest scaffold length (bp) & $16,503,592$ \\
Scaffold L50 & 7 \\
Scaffold N50 length (bp) & $14,943,599$ \\
GC content (\%) & 36.02 \\
Repeat content (\%) & 41.94 \\
Number of predicted genes & 25,813 \\
Average coding sequence length (bp) & 241 \\
Average gene length (bp) & 2431 \\
Number of exons & 140,984 \\
Average number of exons per gene & 5.46 \\
\hline
\end{tabular}

These results are largely consistent with the patterns of autopolyploids, which usually show a few instances of biased gene retention and no genome dominance.

\section{Recent WGD event in L. maritima}

To identify possible WGD events, we calculated the Ks values between the collinear genes. The L. maritima collinear blocks produced two visible peaks, at 0.583 and 1.287 (Fig. 3a), representing two different WGD events. We then estimated the occurrence times of each WGD event based on the Ks values. However, dating ancestral events in plants can be influenced by divergent evolutionary rates ${ }^{46}$. Thus, by aligning the L. maritima peak with the corresponding location in the C. rubella Ks distribution, as in a previous report ${ }^{46}$, we performed evolutionary rate correction (Fig. 3b). After correction, the peaks of Ks for the two WGD events were 0.378 and 0.855 , corresponding to 22.99 and $52.01 \mathrm{Mya}$, respectively (Fig. 3b). The results indicated an ancient WGD event shared with C. rubella and a recent species-specific WGD event in L. maritima. In addition, Ks estimation indicated that C. rubella and L. maritima diverged approximately 21.53 Mya (Fig. 3b). These findings were consistent with those of the synteny and collinearity analyses of $L$. maritima and C. rubella and suggested that L. maritima

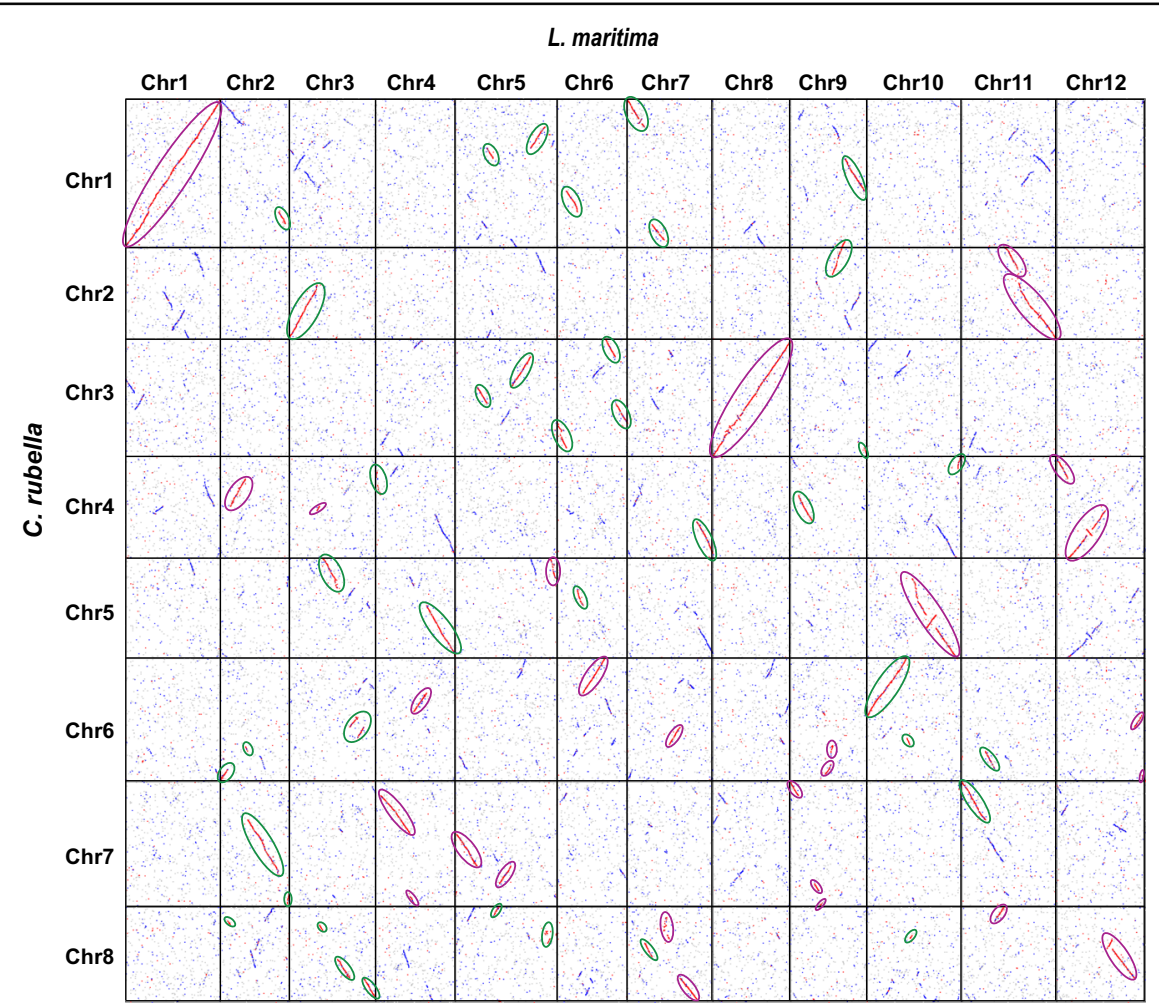

Fig. 2 Dotplot comparing the L. maritima and C. rubella genomes. Collinear regions in the L. maritima genome. Regions from putative subgenomes are circled in purple and green, respectively 

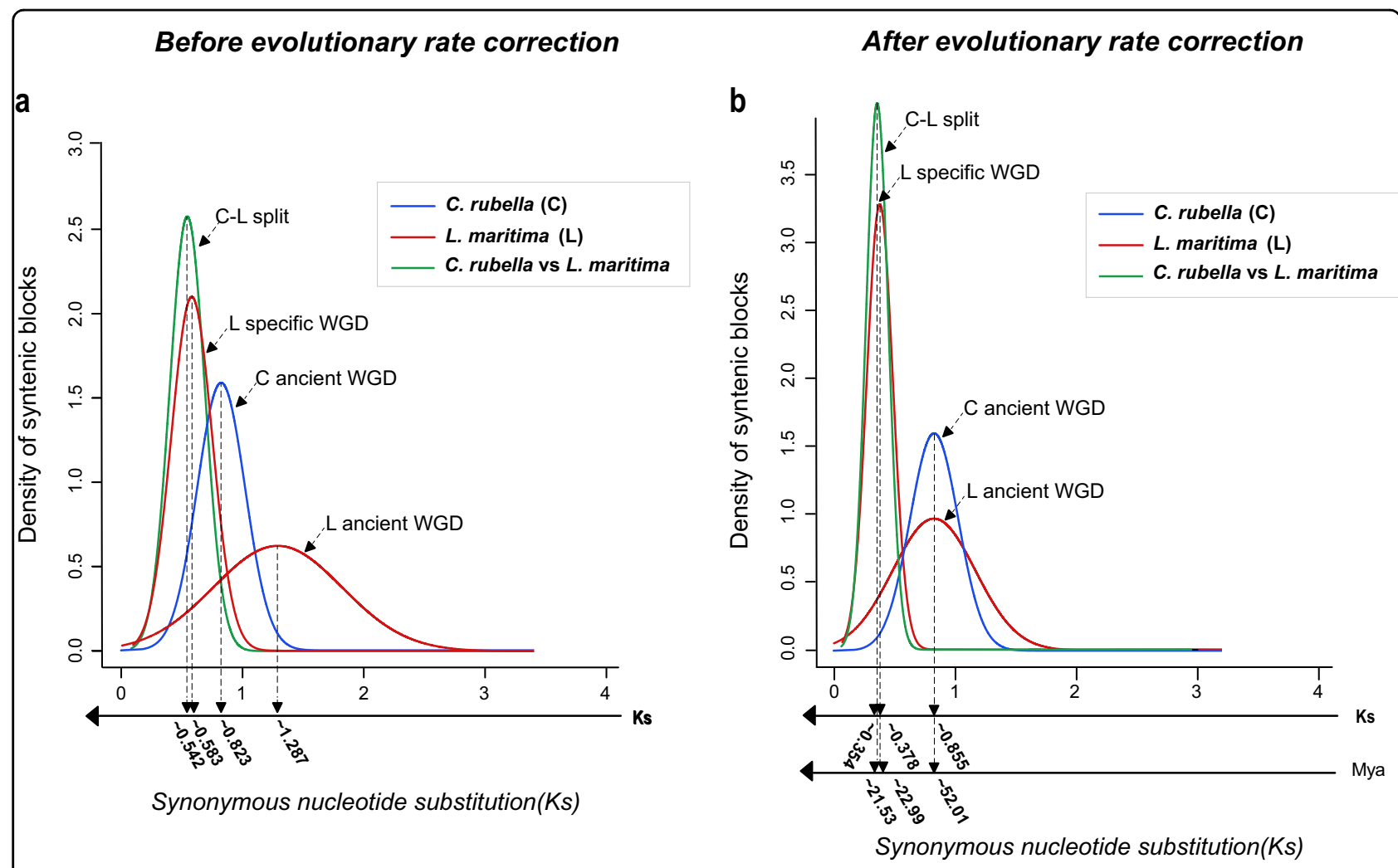

Fig. 3 Evolutionary rate correction. a Distribution of uncorrected Ks values in syntenic blocks; $\mathbf{b}$ distribution of corrected Ks values in syntenic blocks and age estimates for the events

experienced a species-specific WGD event after sharing a WGD event with other Brassicaceae species.

\section{Phylogeny and divergence}

We obtained the genome sequences of representative Brassicaceae species to clarify the genome evolution and divergence of $L$. maritima. Gene family clusters were defined based on the L. maritima protein-coding genes and the annotated gene sets of 10 published genomes (Supplementary Table 9) using OrthoFinder v2.3.12 $2^{47}$ A total of 25,316 orthogroups were determined across the 11 species. Among these orthogroups, 1,986 were putative single-copy gene families, and 24,705 genes from $L$. maritima could be clustered into 16,821 orthogroups. In addition, we identified 1878 L. maritima-specific genes in these gene families. Functional annotations of these genes indicated that they were distinctly enriched in the GO terms "positive regulation of response to salt stress", "abscisic acid-activated signaling pathway", "response to freezing", "response to stimulus", and "response to biotic stimulus", indicating that the genes retained after the WGD event may be relevant in the adaptation of $L$. maritima to multiple environmental stress factors (Fig. 4a and Supplementary Tables 10 and 11). For example, a homolog of these L. maritima-specific genes, $A B I 4$, acts as both an activator and a repressor of gene expression and plays a critical role in phytohormone signaling pathways in plant development and biotic/abiotic stress respon$\operatorname{ses}^{48}$. Another homolog, $A B I 1$, serves as a key repressor of the abscisic acid (ABA) signaling pathway and regulates diverse ABA responses to abiotic stress ${ }^{49,50}$. The speciesspecific calcium-dependent protein kinase (CDPK) genes recovered here (Supplementary Table 11) were also demonstrated to be involved in numerous aspects of plant growth and development, from sensing biotic and abiotic stress to mediating hormone-related development ${ }^{51}$.

To verify the phylogenetic position of $L$. maritima, we used the concatenated protein sequence alignment of the 1986 single-copy gene families in the 11-species phylogenetic analyses. The results confirmed that $L$. maritima belonged to Lineage $\mathrm{II}^{11,52}$ (Fig. $4 \mathrm{~b}$ ), consistent with its position in the chloroplast genome phylogeny reported previously $^{53}$. In our analyses performed using MCMCtree $^{54}$, L. maritima was estimated to have diverged from the other closely related species $\sim 22.63$ (18.74, 26.61) Mya (Fig. 4b).

\section{Expansion and contraction of gene families in L. maritima}

Gene families with significantly expanded or contracted copy numbers are usually related to the adaptive 


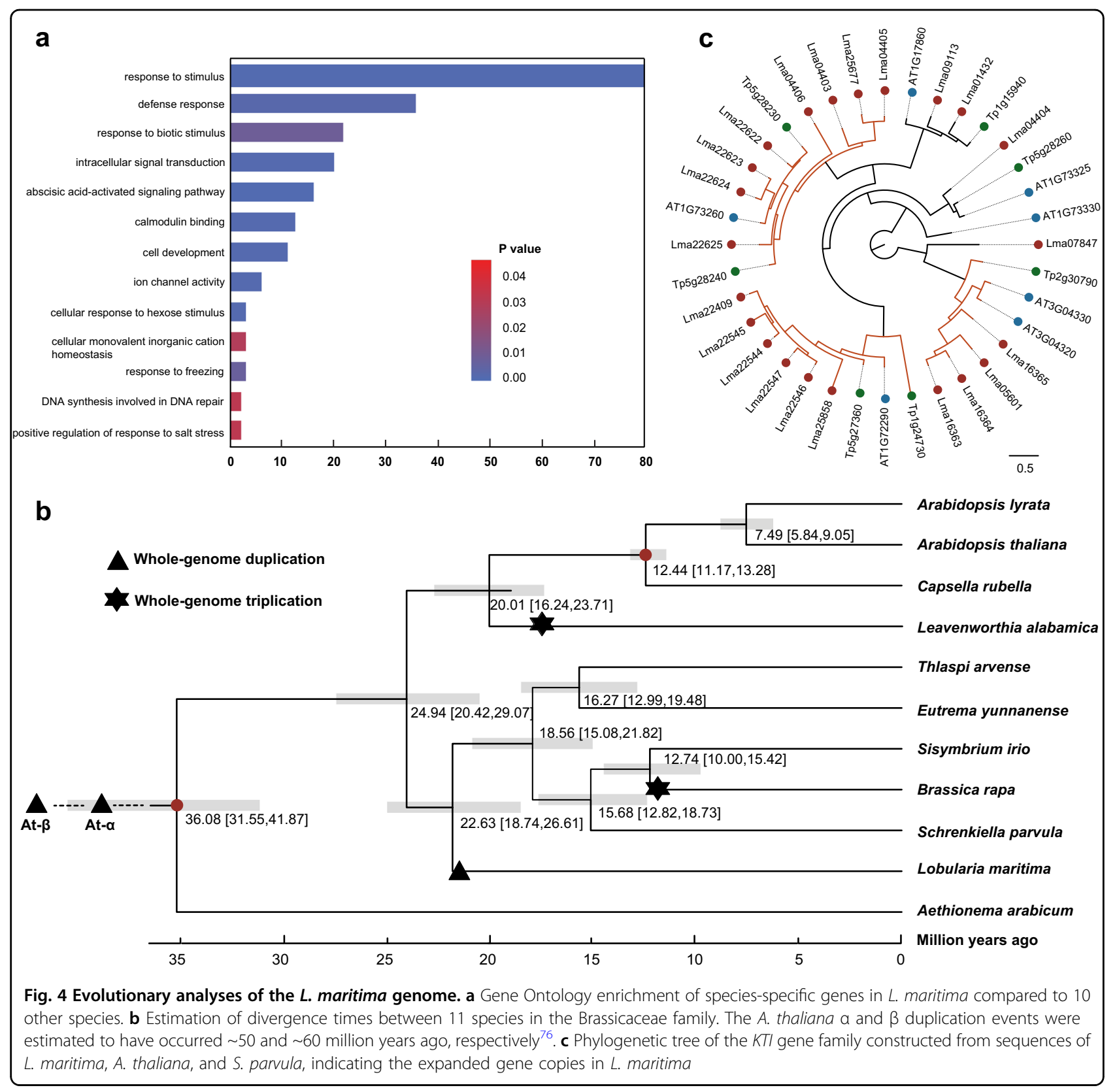

divergence of one species from closely related species ${ }^{55,56}$. We compared the genomes of L. maritima and 10 other species, with Aethionema arabicum as the outgroup (Fig. $4 \mathrm{~b})$, to explore the expansion and contraction of the gene families in L. maritima. Twenty-five gene families, comprising 319 genes, were significantly expanded in $L$. maritima $(P<0.05)$. Functional annotation of these genes indicated that they were mainly enriched in "response to molecule of bacterial origin", "response to insect", "response to molecule of fungal origin", "response to wounding" and "response to salt stress" (Supplementary Tables 12 and 13). For example, one of the expanded gene families, the KTI gene family, comprised versatile protease inhibitors related to defense against insect attack (Fig. $4 c)^{57}$. In addition, the HIPP gene family, involved in stress responses $^{58}$, was also greatly expanded in L. maritima.

\section{Positively selected genes in L. maritima}

Genes with signs of positive selection are usually regarded to be involved in the adaptive divergence of one species from closely related species ${ }^{59}$. We conducted positive selection analysis by using $L$. maritima as the foreground branch and five related Brassicaceae species (Eutrema yunnanense, C. rubella, A. arabicum, A. lyrata, and Schrenkiella parvula) as the background branches. We identified 10,581 single-copy orthologous gene 


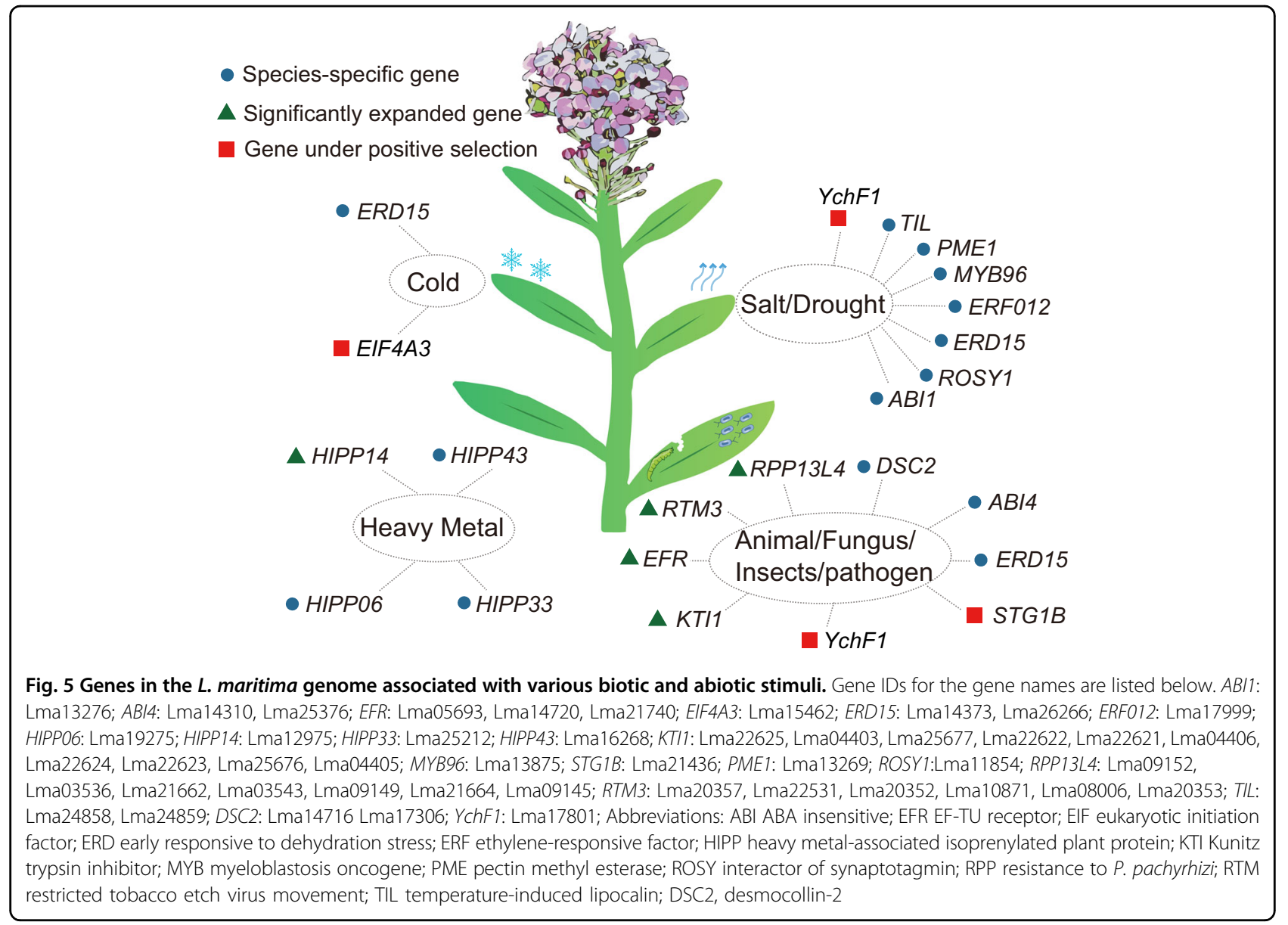

families. To identify the genes that evolved in response to positive selection, we adopted the branch-site model in the PAML v4.9 package $^{54}$. After false discovery rate (FDR) correction, we identified 50 genes that were possibly under positive selection. The functions of the significantly positively selected genes (PSGs) indicated that they were associated with stress tolerance and the survival of plants (Fig. 5 and Supplementary Table 14). For example, one of the genes was $S G T 1 B$, which was found to be involved in innate immunity and resistance in plants mediated by multiple $\mathrm{R}$ genes ${ }^{60-63}$. Another of the genes was $Y c h F 1$, which is involved in salinity stress tolerance and disease resistance against bacterial pathogens ${ }^{64}$. Another of the genes, EIF4A3, is an important factor for abiotic stress adaptation, which can regulate plant resistance to abiotic stress partially by regulating the expression of acetoacetylCoA thiolase $2^{65}$.

\section{Discussion}

L. maritima is an important ornamental plant in horticulture because of its colorful flowers and stress tolerance. In this study, by combining Illumina and $\mathrm{Hi}-\mathrm{C}$ data, a chromosome-level high-quality $L$. maritima genome was assembled. The L. maritima genome was $\sim 197.70 \mathrm{Mb}$ in size, and $88.31 \%(174.59 \mathrm{Mb})$ of the sequences were assigned to 12 pseudochromosomes. We annotated 25,813 genes and found substantially more repetitive elements (especially intact LTR retrotransposons) in the L. maritima genome than in the genomes of other Brassicaceae species. In addition, most intact LTR retrotransposons expanded rapidly in the recent past. Such proliferation of LTR retrotransposons may have partly resulted in the increased genome size of L. maritima. Phylogenetic reconstructions showed that L. maritima diverged early as an independent branch of Brassicaceae Lineage II.

In the histories of many diverse eukaryotes, including Danio rerio ${ }^{66}$, Saccharomyces cerevisiae ${ }^{67}$, and A. thali$a n a^{68-71}$, WGDs have been discovered. Through large-scale phylogenomic analyses, ancient WGDs were found to occur in the common ancestors of both seed plants and angiosperms $^{4,9,71,72}$. WGDs have played an essential role in angiosperm diversification and environmental adaptation ${ }^{9}$. Polyploids can tolerate high environmental stress, with present-day polyploids often appearing to occur at high frequencies in disturbed and harsh environments ${ }^{73-75}$. 
Under environmental stresses, polyploids may have been more successful because their changing environments created many opportunities to make use of the evolutionary benefits of WGDs ${ }^{76}$. The comparison of L. maritima and the diploid C. rubella indicated a recent WGD event that was specific to L. maritima, followed by extensive chromosomal rearrangements. Furthermore, we evaluated whether biased gene retention occurred after the WGD event. Two subgenomes retained a similar number of genes. However, neither subgenome showed genome dominance. This indicates that L. maritima might have undergone an autopolyploidization event. Analysis of the Ks values between the collinear genes suggested that the recent $L$. maritima-specific WGD event occurred 22.99 Mya. The comparison of between-species Ks distributions indicated that the L. maritima-C. rubella divergence occurred $\sim 21.53$ Mya. Thus, this divergence and the aforementioned $L$. maritima-specific WGD event occurred at almost the same time. L. maritima and C. rubella belong to two major lineages, and it is highly likely that the divergence of the two major lineages and genus diversification of each lineage in Brassicaceae occurred radiatively at the same time. This rapid radiation was accompanied by polyploidy in a few of the genera. This is also consistent with the previous suggestion that further WGDs might have occurred in Brassicaceae since the Neogene, with radiative diversification, which further helped members of this family colonize arid habitats by increasing their stress tolerance ${ }^{26}$. As a result of the WGD event, species-specific genes and expanded gene families become further involved in responses to environmental stresses, for example, drought and pathogen attack, which might have facilitated the adaptation of $L$. maritima to harsh environments. In addition, the positively selected genes in L. maritima may have increased defense against fungal and bacterial attack. Thus, the species-specific WGD event may have promoted the adaptation of L. maritima to harsh environments, which is consistent with previous findings for numerous plants ${ }^{76,77}$. These genomic traits may also explain why L. maritima is a nickel hyperaccumulator $^{32}$ and a halophyte with a high tolerance to salt stress $^{33}$. Overall, whole-genome sequencing of L. maritima could elucidate the stress tolerance of this ornamental plant and be useful in future breeding programs.

\section{Materials and methods}

\section{Materials and DNA/RNA extraction}

The L. maritima seedling was cultivated in Jinjiang District, Chengdu City, Sichuan Province, China (N 30 $34^{\prime}$ $21.86^{\prime \prime}$, E $\left.104^{\circ} 09^{\prime} 45.47^{\prime \prime}\right)$. We harvested fresh and healthy roots, stems, leaves and flowers and immediately froze them in liquid nitrogen. Before DNA/RNA extraction, we stored these tissues in a $-80^{\circ} \mathrm{C}$ freezer in the laboratory. To extract high-quality genomic DNA, the cetyl trimethylammonium bromide $(\mathrm{CTAB})^{78}$ method was used.
Additionally, we extracted total RNA from the flower, stem and leaf tissues using Qiagen RNeasy Plant Mini Kits.

\section{Library construction and sequencing}

We randomly fragmented the purified genomic DNA using a focused ultrasonicator and obtained fragments of desired lengths by electrophoresing the DNA fragments in 0.8\% General Purpose Agarose E-Gel. Then, we created Illumina libraries with large (2-, 5-, 10- and 20-kb) and small (350- and 500-bp) inserts using the purified DNA fragments. Based on the PE-150 protocol, the libraries were finally sequenced on an Illumina HiSeq 2000 platform. RNA libraries were constructed with a TruSeq RNA Library Preparation Kit v2 and sequenced on the same platform.

A Hi-C library was constructed using five main steps. First, we fixed the sample with formaldehyde and crosslinked DNA-DNA interactions that are bridged by proteins. Second, the crosslinked DNA was treated with the restriction endonuclease Hind III to produce sticky ends. Third, terminal DNA repair was used to introduce biotinlabeled bases in order to facilitate subsequent DNA purification and capture. Next, we ensured the location of the interacting DNA through cyclization of the end-repaired DNA and DNA fragments. Finally, we extracted and purified the DNA sample and then used Covaris S2 to shear the DNA sample. After A-tailing, pulldown, and adapter ligation, the DNA library was sequenced on an Illumina platform using the PE-150 protocol. We used HiCPro v2.8.1 $1^{79}$ to remove duplicates and then assessed quality. After trimming low-quality reads and removing adapters, more than $22.31 \mathrm{~Gb}(\sim 112.89$-fold coverage) of clean data was generated. Then, all clean data were submitted to the 3D-DNA v180419 pipeline ${ }^{80}$.

\section{Genome assembly}

Approximately $79.49 \mathrm{~Gb}$ of raw reads was generated by sequencing all six DNA libraries. These raw reads were filtered following a previous study ${ }^{81}$. We first used Trimmomatic $\mathrm{v} 0.33^{82}$ to perform quality filtering of short reads. We then used the BFC error corrector ${ }^{83}$ followed by FastUniq v1.1 ${ }^{84}$ to delete duplicates in the mate pair data. The resultant reads produced approximately 59.77 Gb of clean data (Supplementary Table 1).

We used Platanus v1.2.4 ${ }^{85}$ software to perform de novo assembly of the L. maritima genome. Thereafter, using the 3D-DNA v180419 pipeline ${ }^{80}$, the draft assembly was scaffolded with the $\mathrm{Hi}-\mathrm{C}$ clean reads. Using the Juicer v1.6.2 pipeline ${ }^{86}$, we aligned the $\mathrm{Hi}-\mathrm{C}$ clean reads to the draft assembly genome. We then used Juicebox Assembly Tools $^{87}$ to polish the results from the 3D-DNA v180419 pipeline. The $\mathrm{Hi}-\mathrm{C}$ scaffolding was anchored on 12 pseudochromosomes. In total, $88.31 \%$ of the assembled 
sequences were related to the pseudochromosomes. In addition, we assessed the quality of the assembled genome using the BUSCO v4.1.2 ${ }^{35}$ pipeline (database: embryophyta odb10, 2020-09-02, containing 1,614 BUSCO genes).

\section{Repeat element annotation}

Repeat elements were identified with the RepeatMasker v4.0.7 $7^{88}$ and RepeatModeler v1.0.11 ${ }^{89}$ programs using the assembled $L$. maritima genome as the input. We also identified intact LTR retrotransposons by searching the $L$. maritima genome using LTRharvest $\mathrm{v} 1.5 .10^{90}$ and LTR_Finder $v 1.06^{91}$. We further combined these results using LTR_retriever v1. $9^{92}$. We also estimated insertion time according to a substitution rate of $7 \times 10^{-9} /$ site/year.

\section{Gene prediction and annotation}

To predict genes in the L. maritima genome, we first assembled transcripts using the de novo and genomeguided modes in Trinity v2.6.6 ${ }^{93}$. Then, these transcripts were used to create transcript-based predictions with the PASA v2.1.0 pipeline ${ }^{94}$. We also carried out homolog predictions. In such predictions, the protein sequences of A. thaliana, A arabicum, A. lyrata, Eutrema yunnanense, Brassica rapa, Sisymbrium irio, C. rubella, Tarenaya hassleriana, Leavenworthia alabamica and Carica papaya were mapped to the L. maritima genome using Exonerate v2.2.0 (https://www.ebi.ac.uk/about/vertebrategenomics/software/exonerate). GlimmerHMM v3.0.4 ${ }^{95}$ and Augustus v3.2.2 ${ }^{96}$ were trained with genes from the PASA results and used for de novo gene prediction. We merged the gene models from the three sources using EVidenceModeler v1.1.1 ${ }^{97}$. To annotate the functions of all predicted genes, we aligned the protein sequences of $L$. maritima to Swiss-Prot and TrEMBL ${ }^{36}$ using blastp and generated functional assignments based on the best hit. Protein domains were determined by searching against the InterPro ${ }^{37}$ database. In addition, Blast $2 \mathrm{GO}$ v2.5 $.5^{98}$ was used to identify the Gene Ontology ${ }^{38}$ annotations and $\mathrm{KEGG}^{39}$ pathways using the KAAS server (https://www. genome.jp/kegg/kaas).

\section{Synteny and WGD}

To construct syntenic blocks between $L$. maritima and $C$. rubella, all protein sequences of L. maritima were compared to protein sequences of $C$. rubella. The gene pairs with an $e$-value $\leq 1 \mathrm{e}-5$ were further analyzed. We applied the ColinearScan v1.0.1 $1^{41}$ program, which can effectively evaluate genomic blocks of collinear genes, and the MCScanX v1 package ${ }^{42}$ to find the syntenic blocks between the C. rubella and L. maritima genomes. Thereafter, we used these collinear gene pairs to construct a dotplot. Next, we used the script "add_ka_and_ks_to_collinearity.pl" in MCScanX to calculate the Ks values of the collinear orthologous gene pairs. We converted the Ks values to divergence times $(\mathrm{T})$ based on $T=K \mathrm{~s} / 2 r$, where $\mathrm{r}$ is the neutral substitution rate $\left(8.22 \times 10^{-9}\right)$. Finally, we performed evolutionary rate correction because of the inconsistent evolutionary rates among species. The evolutionary rate correction method was as reported by Wang et al. ${ }^{46}$. Briefly, under the assumption that the C. rubella peak appears at $k_{C}$ and the L. maritima peak appears at $k_{L}$, we can use the equation $r=\left(k_{L}-k_{C}\right) / k_{C}$ to describe the relative evolutionary rate of $L$. maritima. Then, rate correction was performed to discover the corrected rate $k_{L}$ correction of $L$. maritima relative to $k_{C}$ : (1) For the Ks between duplicates in L. maritima, we defined the correction coefficient $W_{L}$ as $k_{\mathrm{L}}$ correction $/ k_{L}=k_{C} / k_{L}=W_{L}$; thus, we obtained $k_{\mathrm{L}}$ correction $=k_{C} / k_{L} \times k_{L}=1 /(1+\mathrm{r}) \times k_{L}$ and $W_{L}=1 /(1+\mathrm{r})$. (2) For the Ks between homologous genes from $C$. rubella and $L$. maritima, if the peak was located at $k_{L-C}$, supposing the correction coefficient $W_{L}$ in L. maritima, we then calculated a corrected evolutionary rate $k_{\mathrm{L}-\mathrm{C} \text {-correction }}=W_{L} \times \mathrm{k}_{L-C}$.

\section{Phylogeny and divergence}

The genomes of $L$. maritima and 10 other species $(A$. arabicum, B. rapa, L. alabamica, E. yunnanense, S. irio, $A$. thaliana, A. lyrata, C. rubella, S. parvula and Thlaspi arvense) were selected to generate clusters of gene families. We retained only the longest protein sequence. We removed redundant sequences based on alternative splicing variations. Using OrthoFinder v2.3.12 ${ }^{47}$, we obtained orthologous gene families. Protein sequences from 1986 single-copy gene families were used to construct a phylogenetic tree. MAFFT v7.313 ${ }^{99}$ software was used for sequence alignment of each single-copy gene family with default settings. A phylogenetic tree was built using RAxML v8.0.0 $0^{100}$ under the PROTGAMMALGX model, and divergence times were calculated using the MCMCTree program of the PAML v4.9 package ${ }^{54}$. The calibration information for MCMCTree was extracted based on the TimeTree database ${ }^{101}$ (http://www.time.org/).

\section{Gene family expansion and contraction}

Based on the dated phylogeny, we determined the expansions and contractions of orthologous gene families in the 11 Brassicaceae species (A. arabicum, B. rapa, $L$. alabamica, E. yunnanense, S. irio, T. arvense, C. rubella, A. thaliana, A. lyrata, S. parvula, and L. maritima) by using the CAFÉ v4.2 $2^{102}$ program. Genes in significantly expanded families were then used for Gene Ontology enrichment analysis.

\section{Genes under positive selection}

We selected six genomes, i.e., those of $A$. arabicum, $A$. lyrata, C. rubella, E. yunnanense, S. parvula and L. maritima, to identify orthologs for analyzing positive selection. First, Proteinortho v6.0.21 $1^{103}$ was used to detect 
orthologs among the six genomes. Next, we used the PosiGene v0.1 $1^{104}$ pipeline for genome-wide detection of the genes with positive selection and specified the $L$. maritima clade as the foreground branch. Finally, PSGs were identified based on an FDR-corrected $P$ value $<0.05$.

\section{Acknowledgements}

This work was supported by the National Key Research and Development Program of China (2017YFC0505203), National Natural Science Foundation of China (31590821), Fundamental Research Funds for the Central Universities (2018CDDY-S02-SCU and SCU2019D013), National High-Level Talents Special Support Plan (10 Thousand of People Plan), and 985 and 211 Projects of Sichuan University.

\section{Author contributions}

Q.H., J.L., and Z.X. designed the research; L.Z. collected the materials and performed genome sequencing; L.H., Y.M., W.Y., T.L., J.J., L.W., and L.F. conducted the genome assembly, annotation and evolution-related data analysis; L.H., Q.H., and J.L. wrote the paper. All authors read and approved the final paper.

\section{Data availability}

Raw Illumina-short reads and $\mathrm{Hi}-\mathrm{C}$ reads used for de novo whole-genome assembly have been deposited in the National Center for Biotechnology Information (NCBI) Sequence Read Archive database under accession number PRJNA630530. The genome and related annotation data have been deposited in the National Genomics Data Center (PRJCA002888).

\section{Conflict of interest}

The authors declare that they have no conflict of interest.

\section{Publisher's note}

Springer Nature remains neutral with regard to jurisdictional claims in published maps and institutional affiliations.

Supplementary Information accompanies this paper at (https://doi.org/ 10.1038/s41438-020-00422-w).

Received: 9 April 2020 Revised: 21 September 2020 Accepted:

30 September 2020

Published online: 01 December 2020

\section{References}

1. Schranz, M. E. \& Mitchell-Olds, T. Independent ancient polyploidy events in the sister families Brassicaceae and Cleomaceae. Plant Cell. 18, 1152-1165 (2006).

2. Van de Peer, Y., Mizrachi, E. \& Marchal, K. The evolutionary significance of polyploidy. Nat. Rev. Genet. 18, 411-424 (2017).

3. Adams, K. L. \& Wendel, J. F. Polyploidy and genome evolution in plants. Curr. Opin. Plant Biol. 8, 135-141 (2005).

4. Blanc, G. \& Wolfe, K. H. Widespread paleopolyploidy in model plant species inferred from age distributions of duplicate genes. Plant Cell. 16, 1667-1678 (2004).

5. Paterson, A. H., Bowers, J. E. \& Chapman, B. A. Ancient polyploidization predating divergence of the cereals, and its consequences for comparative genomics. Proc. Natl Acad. Sci. USA 101, 9903-9908 (2004).

6. Bertioli, D. J. et al. An analysis of synteny of Arachis with Lotus and Medicago sheds new light on the structure, stability and evolution of legume genomes. BMC Genomics. 10, 45 (2009).

7. Tang, H., Bowers, J. E., Wang, X. \& Paterson, A. H. Angiosperm genome comparisons reveal early polyploidy in the monocot lineage. Proc. Natl Acad. Sci. USA 107, 472-477 (2010).

8. Jiao, Y., Li, J., Tang, H. \& Paterson, A. H. Integrated syntenic and phylogenomic analyses reveal an ancient genome duplication in monocots. Plant Cell. 26, 2792-2802 (2014).
9. Jiao, Y. et al. Ancestral polyploidy in seed plants and angiosperms. Nature 473, 97-100 (2011).

10. Cai, J. et al. The genome sequence of the orchid Phalaenopsis equestris. Nat. Genet. 47, 65 (2015)

11. Huang, C. et al. Resolution of Brassicaceae phylogeny using nuclear genes uncovers nested radiations and supports convergent morphological evolution. Mol. Biol. Evol. 33, 394-412 (2016).

12. Hegarty, M. J. \& Hiscock, S. J. Genomic clues to the evolutionary success of polyploid plants. Curr. Biol. 18, R435-R444 (2008).

13. Sémon, M. \& Wolfe, K. H. Reciprocal gene loss between Tetraodon and zebrafish after whole genome duplication in their ancestor. Trends Genet. 23, 108-112 (2007).

14. Perumal, S. et al. Elucidating the major hidden genomic components of the $A, C$, and $A C$ genomes and their influence on Brassica evolution. Sci. Rep. 7, 1-12 (2017).

15. Kiefer, M. et al. BrassiBase: introduction to a novel knowledge database on Brassicaceae evolution. Plant Cell Physiol. 55, e3 (2014).

16. Appel, O. \& Al-Shehbaz, I. A. Cruciferae. in Flowering Plants-Dicotyledons (Springer, 2003).

17. Al-Shehbaz, I. A., Beilstein, M. A. \& Kellogg, E. A. Systematics and phylogeny of the Brassicaceae (Cruciferae): an overview. Plant Syst. Evol. 259, 89-120 (2006).

18. O'Kane Jr, S. L. Brassicaceae, molecular systematics and evolution of. Brenner's Encycl. Genet. Second Ed. 374-376, https://doi.org/10.1016/B978-0-12-3749840.00169-8 (2013).

19. Blanc, G., Hokamp, K. \& Wolfe, K. H. A recent polyploidy superimposed on older large-scale duplications in the Arabidopsis genome. Genome Res. 13, 137-144 (2003).

20. De Bodt, S., Maere, S. \& de Peer, Y. Genome duplication and the origin of angiosperms. Trends Ecol. Evol. 20, 591-597 (2005).

21. Soltis, D. E. et al. Polyploidy and angiosperm diversification. Am. J. Bot. 96 336-348 (2009).

22. Ming, R. et al. The draft genome of the transgenic tropical fruit tree papaya (Carica papaya Linnaeus). Nature 452, 991-996 (2008).

23. Tang, H. et al. Synteny and collinearity in plant genomes. Science $\mathbf{3 2 0}$ 486-488 (2008).

24. Fawcett, J. A., Maere, S. \& Van de Peer, Y. Plants with double genomes might have had a better chance to survive the Cretaceous-Tertiary extinction event. Proc. Natl Acad. Sci. USA 106, 5737-5742 (2009).

25. Wang, X. et al. The genome of the mesopolyploid crop species Brassica rapa. Nat. Genet. 43, 1035 (2011).

26. Kagale, S. et al. Polyploid Evolution of the Brassicaceae during the Cenozoic Era. Plant Cell. 26, 2777-2791 (2014).

27. Guo, X. et al. The genomes of two Eutrema species provide insight into plant adaptation to high altitudes. DNA Res. 25, 307-315 (2018).

28. Kang, M. et al. A chromosome-scale genome assembly of Isatis indigotica, an important medicinal plant used in traditional Chinese medicine. Hortic. Res. 7, 1-10 (2020).

29. Huang, R. et al. Artificially induced polyploidization in Lobularia maritima (L.) Desv. and its effect on morphological traits. Hortscience 50, 636-639 (2015).

30. Gómez, J. M. et al. Phenotypic selection and response to selection in Lobularia maritima: importance of direct and correlational components of natural selection. J. Evol. Biol. 13, 689-699 (2000).

31. Polunin, O. \& Everard, B. Flowers of Europe: A Field Guide (Oxford University Press, Oxford, 1969).

32. Yuan, X. Y., Zhang, X. Y., Ma, J. \& Hou, X. F. Tissue culture in vitro and establishment of regeneration system of Lobularia maritima. North. Hort. $\mathbf{8}$, 145-146 (2010).

33. Popova, O. V. \& Golldack, D. In the halotolerant Lobularia maritima (Brassicaceae) salt adaptation correlates with activation of the vacuolar H+-ATPase and the vacuolar $\mathrm{Na}+/ \mathrm{H}+$ antiporter. J. Plant Physiol. 164, 1278-1288 (2007).

34. Liu, B. et al. Estimation of genomic characteristics by analyzing k-mer frequency in de novo genome projects. Quant. Biol. 35, 62-67 (2013).

35. Simão, F. A., Waterhouse, R. M., loannidis, P., Kriventseva, E. V. \& Zdobnov, E. M. BUSCO: assessing genome assembly and annotation completeness with single-copy orthologs. Bioinformatics 31, 3210-3212 (2015).

36. Bairoch, A. \& Apweiler, R. The SWISS-PROT protein sequence database and its supplement TrEMBL in 2000. Nucleic Acids Res. 28, 45-48 (2000).

37. Hunter, S. et al. InterPro: the integrative protein signature database. Nucleic Acids Res. 37, D211-D215 (2008).

38. Ashburner, M. et al. Gene ontology: tool for the unification of biology. Nat. Genet. 25, 25 (2000). 
39. Kanehisa, M. \& Goto, S. KEGG: Kyoto encyclopedia of genes and genomes. Nucleic Acids Res. 28, 27-30 (2000).

40. Oliver, K. R., McComb, J. A. \& Greene, W. K. Transposable elements: powerful contributors to angiosperm evolution and diversity. Genome Biol. Evol. $\mathbf{5}$ 1886-1901 (2013).

41. Wang, $X$. et al. Statistical inference of chromosomal homology based on gene colinearity and applications to Arabidopsis and rice. BMC Bioinforma. 7, 447 (2006).

42. Wang, Y. et al. MCScanX: a toolkit for detection and evolutionary analysis of gene synteny and collinearity. Nucleic Acids Res. 40, e49 (2012).

43. Lysak, M. A., Mandáková, T. \& Schranz, M. E. Comparative paleogenomics of crucifers: ancestral genomic blocks revisited. Curr. Opin. Plant Biol. 30, 108-115 (2016).

44. Mandáková, T., Guo, X., Özüdoğru, B., Mummenhoff, K. \& Lysak, M. A. Hybridization-facilitated genome merger and repeated chromosome fusion after 8 million years. Plant J. 96, 748-760 (2018).

45. Mandáková, T. \& Lysak, M. A. Chromosomal phylogeny and karyotype evolution in $x=7$ crucifer species (Brassicaceae). Plant Cell. 20, 2559-2570 (2008).

46. Wang, J. et al. Two likely auto-tetraploidization events shaped kiwifruit genome and contributed to establishment of the Actinidiaceae family. iscience 7, 230-240 (2018).

47. Emms, D. M. \& Kelly, S. OrthoFinder: solving fundamental biases in whole genome comparisons dramatically improves orthogroup inference accuracy. Genome Biol. 16, 157 (2015).

48. Chandrasekaran, U., Luo, X., Zhou, W. \& Shu, K. Multifaceted signaling networks mediated by abscisic acid insensitive 4. Plant Commun. 1, 100040 (2020)

49. Harb, A., Krishnan, A., Ambavaram, M. M. R. \& Pereira, A. Molecular and physiological aanalysis of drought stress in Arabidopsis reveals early responses leading to acclimation in plant growth. Plant Physiol. 154, 1254-1271 (2010).

50. Kariola, T. et al. Early responsive to dehydration 15, a negative regulator of abscisic acid responses in Arabidopsis. Plant Physiol. 142, 1559-1573 (2006).

51. Boudsocq, M. \& Sheen, J. CDPKs in immune and stress signaling. Trends Plant Sci. 18, 30-40 (2013).

52. Beilstein, M. A., Al-Shehbaz, I. A. \& Kellogg, E. A. Brassicaceae phylogeny and trichome evolution. Am. J. Bot. 93, 607-619 (2006).

53. Guo, X. et al. Plastome phylogeny and early diversification of Brassicaceae. BMC Genomics. 18, 176 (2017).

54. Yang, Z. PAML 4: phylogenetic analysis by maximum likelihood. Mol. Biol. Evol. 24, 1586-1591 (2007).

55. Dassanayake, M. et al. The genome of the extremophile crucifer Thellungiella parvula. Nat. Genet. 43, 913-918 (2011).

56. Sudmant, P. H. et al. Diversity of human copy number variation and multicopy genes. Science 330, 641-646 (2010).

57. Arnaiz, A. et al. Arabidopsis kunitz trypsin inhibitors in defense against spider mites. Front. Plant Sci. 9, 986 (2018).

58. Zschiesche, W. et al. The zinc-binding nuclear protein HIPP3 acts as an upstream regulator of the salicylate-dependent plant immunity pathway and of flowering time in Arabidopsis thaliana. N. Phytol. 207, 1084-1096 (2015).

59. Fitch, W. M. Distinguishing homologous from analogous proteins. Syst. Zool. 19, 99-113 (1970).

60. Azevedo, C. et al. Role of SGT1 in resistance protein accumulation in plant immunity. EMBO J. 25, 2007-2016 (2006).

61. Tör, M. et al. Arabidopsis SGT1b is required for defense signaling conferred by several downy mildew resistance genes. Plant Cell. 14, 993-1003 (2002).

62. Holt, B. F. Antagonistic control of disease resistance protein stability in the plant immune system. Science 309, 929-932 (2005).

63. Austin, M. J. Regulatory role of SGT1 in early R gene-mediated plant defenses. Science 295, 2077-2080 (2002).

64. Cheung, M.Y. et al. ATP binding by the P-loop NTPase OsYchF1 (an unconventional $G$ protein) contributes to biotic but not abiotic stress responses. Proc. Natl Acad. Sci. USA 113, 2648-2653 (2016).

65. Pascuan, C., Frare, R., Alleva, K., Ayub, N. D. \& Soto, G. mRNA biogenesisrelated helicase elF4Alll from Arabidopsis thaliana is an important factor for abiotic stress adaptation. Plant Cell Rep. 35, 1205-1208 (2016).

66. Postlethwait, J. H. et al. Zebrafish comparative genomics and the origins of vertebrate chromosomes. Genome Res. 10, 1890-1902 (2000).

67. Wolfe, K. H. \& Shields, D. C. Molecular evidence for an ancient duplication of the entire yeast genome. Nature 387, 708 (1997)
68. Blanc, G., Barakat, A., Guyot, R., Cooke, R. \& Delseny, M. Extensive duplication and reshuffling in the Arabidopsis genome. Plant Cell. 12, 1093-1101 (2000).

69. Vision, T. J., Brown, D. G. \& Tanksley, S. D. The origins of genomic duplications in Arabidopsis. Science 290, 2114-2117 (2000).

70. Simillion, C., Vandepoele, K., Van Montagu, M. C. E., Zabeau, M. \& Van de Peer, Y. The hidden duplication past of Arabidopsis thaliana. Proc. Natl Acad. Sci. USA 99, 13627-13632 (2002).

71. Bowers, J. E., Chapman, B. A., Rong, J. \& Paterson, A. H. Unravelling angiosperm genome evolution by phylogenetic analysis of chromosomal duplication events. Nature 422, 433-438 (2003).

72. Doyle, J. J. et al. Evolutionary genetics of genome merger and doubling in plants. Annu. Rev. Genet. 42, 443-461 (2008).

73. Madlung, A. Polyploidy and its effect on evolutionary success: old questions revisited with new tools. Heredity 110, 99 (2013).

74. Ramsey, J. Polyploidy and ecological adaptation in wild yarrow. Proc. Natl Acad. Sci. USA 108, 7096-7101 (2011).

75. Diallo, A. M., Nielsen, L. R., Kjær, E. D., Petersen, K. K. \& Ræbild, A. Polyploidy can confer superiority to West African Acacia senegal (L.) Willd. trees. Front. Plant Sci. 7, 821 (2016).

76. Vanneste, K., Baele, G., Maere, S. \& Van de Peer, Y. Analysis of 41 plant genomes supports a wave of successful genome duplications in association with the Cretaceous-Paleogene boundary. Genome Res. 24, 1334-1347 (2014).

77. Vanneste, K., Maere, S. \& Van de Peer, Y. Tangled up in two: a burst of genome duplications at the end of the Cretaceous and the consequences for plant evolution. Philos. Trans. R. Soc. B Biol. Sci. 369, 20130353 (2014).

78. Doyle, J. J. \& Doyle, J. L. A rapid DNA isolation procedure for small quantities of fresh leaf tissue. Phytochem. Bull. 19, 11-15 (1987).

79. Servant, N. et al. HiC-Pro: an optimized and flexible pipeline for $\mathrm{Hi}-\mathrm{C}$ data processing. Genome Biol. 16, 259 (2015).

80. Dudchenko, O. et al. De novo assembly of the Aedes aegypti genome using $\mathrm{Hi}-\mathrm{C}$ yields chromosome-length scaffolds. Science 356, 92-95 (2017).

81. $\mathrm{Wu}, \mathrm{H}$. et al. A high-quality Actinidia chinensis (kiwifruit) genome. Hortic. Res. $\mathbf{6}$, 1-9 (2019)

82. Bolger, A. M., Lohse, M. \& Usadel, B. Trimmomatic: a flexible trimmer for Illumina sequence data. Bioinformatics 30, 2114-2120 (2014).

83. Li, H. BFC: correcting Illumina sequencing errors. Bioinformatics 31, 2885-2887 (2015)

84. $\mathrm{Xu}, \mathrm{H}$. et al. FastUniq: a fast de novo duplicates removal tool for paired short reads. PLoS ONE 7, e52249 (2012)

85. Kajitani, R. et al. Efficient de novo assembly of highly heterozygous genomes from whole-genome shotgun short reads. Genome Res. 24, 1384-1395 (2014).

86. Durand, N. C. et al. Juicer provides a one-click system for analyzing loopresolution Hi-C experiments. Cell Syst. 3, 95-98 (2016).

87. Durand, N. C. et al. Juicebox provides a visualization system for Hi-C contact maps with unlimited zoom. Cell Systems 3, 99-101, https://doi.org/10.1016/j. cels.2015.07.012 (2016).

88. Tarailo-Graovac, M. \& Chen, N. Using RepeatMasker to identify repetitive elements in genomic sequences. In Current Protocols in Bioinformatics (John Wiley \& Sons, Inc., 2002).

89. Jurka, J. et al. Repbase Update, a database of eukaryotic repetitive elements. Cytogenet. Genome Res. 110, 462-467 (2005).

90. Ellinghaus, D., Kurtz, S. \& Willhoeft, U. LTRharvest, an efficient and flexible software for de novo detection of LTR retrotransposons. BMC Bioinform. 9, 18 (2008)

91. Xu, Z. \& Wang, H. LTR_FINDER: an efficient tool for the prediction of fulllength LTR retrotransposons. Nucleic Acids Res. 35, W265-W268 (2007).

92. Ou, S. \& Jiang, N. LTR_retriever: a highly accurate and sensitive program for identification of long terminal repeat retrotransposons. Plant Physiol. 176, 1410-1422 (2018).

93. Haas, B. J. et al. De novo transcript sequence reconstruction from RNA-seq using the Trinity platform for reference generation and analysis. Nat. Protoc 8, 1494-1512 (2013).

94. Haas, B. J. et al. Improving the Arabidopsis genome annotation using maximal transcript alignment assemblies. Nucleic Acids Res. 31, 5654-5666 (2003).

95. Majoros, W. H., Pertea, M. \& Salzberg, S. L. TigrScan and GlimmerHMM: two open source ab initio eukaryotic gene-finders. Bioinformatics 20, 2878-2879 (2004). 
96. Stanke, M., Steinkamp, R., Waack, S. \& Morgenstern, B. AUGUSTUS: a web server for gene finding in eukaryotes. Nucleic Acids Res. 32, W309-W312 (2004)

97. Haas, B. J. et al. Automated eukaryotic gene structure annotation using EVidenceModeler and the program to assemble spliced alignments. Genome Biol. 9, 1-22 (2008).

98. Conesa, A. et al. Blast2GO: a universal tool for annotation, visualization and analysis in functional genomics research. Bioinformatics 21, 3674-3676 (2005).

99. Katoh, K. \& Standley, D. M. MAFFT multiple sequence alignment software version 7: improvements in performance and usability. Mol. Biol. Evol. 30, 772-780 (2013).
100. Stamatakis, A. RAxML version 8: a tool for phylogenetic analysis and postanalysis of large phylogenies. Bioinformatics 30, 1312-1313 (2014).

101. Hedges, S. B., Dudley, J. \& Kumar, S. TimeTree: a public knowledge-base of divergence times among organisms. Bioinformatics 22, 2971-2972 (2006).

102. De Bie, T., Cristianini, N., Demuth, J. P. \& Hahn, M. W. CAFE: a computational tool for the study of gene family evolution. Bioinformatics 22, 1269-1271 (2006).

103. Lechner, M. et al. Proteinortho: detection of (co-)orthologs in large-scale analysis. BMC Bioinform. 12, 124 (2011).

104. Sahm, A., Bens, M., Platzer, M. \& Szafranski, K. PosiGene: automated and easyto-use pipeline for genome-wide detection of positively selected genes. Nucleic Acids Res. 45, e100 (2017). 